Proc. Indian Acad. Sci. (Anim. Sci.), Vol. 94, No. 3, June 1985, pp. 309-324.

(C) Printed in India.

\title{
Evolution of insect sociality-A review of some attempts to test modern theories
}

\author{
RAGHAVENDRA GADAGKAR
}

Centre for Ecological Sciences, Indian Institute of Science, Bangalore 560012, India

\begin{abstract}
An important feature of insect societies is the presence of a sterile worker caste that makes it possible for the fertile queens to produce a large number of offsprings. The mechanism of evolution by natural selection of such sterility and similar, though less extreme, forms of altruism has long been considered as a paradox. In recent years a large body of theoretical ideas has accumulated that purports to explain altruistic behaviour within the framework of the theory of natural selection. With special reference to insect sociality three theories namely kin selection, parental manipulation and mutualism have been suggested. Some attempts have now been made to empirically test the mutually exclusive predictions arising out of these alternative theories. A somewhat different approach to empirically distinguishing between kin selection and parental manipulation is to measure sex-investment ratios. This approach was at one time believed to have provided overwhelming support in favour of the theory of kin selection. It has now been realised that several complicating factors such as local mate competition and multiple mating have to be considered before arriving at appropriate theoretical predictions of the two rival theories. I argue in this paper that rigorous quantitative studies on inter-individual variations in behavioural strategies in primitively eusocial insects constitutes yet another approach that is likely to help in understanding the forces that mould the evolution of insect societies.
\end{abstract}

Keywords. Social insects; kin selection; parental manipulation; mutualism; altruism; quantitative ethology; sex-investment ratios.

\section{Introduction}

Living organisms present a fantastic diversity of structure, function and behaviour unparalleled by anything in man's experience. This diversity has kept generations of biologists so busy in describing and cataloguing life phenomena that biology has sometimes been compared to stamp collecting. It is perhaps no exaggeration to say that Darwin along with Wallace changed this scene and converted biology into a 'Real Science' by his unifying theory of evolution by natural selection. Darwin set the tradition of asking such questions as why do peacocks have those incredible feathers?, why is sickle cell anemia more common in certain parts of Africa?, why do Hanuman Langurs commit infanticide?

\section{The theory of evolution}

The theory of evolution by natural selection is as pretty as it is simple. Living organisms normally produce many more offsprings than can be supported by the environment. This results in intense competition for survival. The individuals in each generation are not all identical but show a whole range of variation in their structure, function and behaviour that leads to differences in efficiencies of survival and reproduction; differences both from one variant to another as well as from one environment to 
another. If these features are inherited, as they commonly are, it follows that in any given environment some kinds of organisms survive and reproduce better than others and thereby come to dominate the population. The variants that dominate in any given environment are often described by biologists as being 'adapted' to or being 'fittest' in that environment and the efficiency of survival and reproduction is called the fitness. Darwin put together a lifetime's experience in natural history to amass evidence for his theory (Darwin 1859). The result was overwhelming but there was one odd fact that did not fall in place. It is a testimony to Darwin's perceptive mind that he did not fail to notice this anamoly.

\section{The puzzle}

Many animals ranging from slime molds to man live in societies of varying degrees of organisation. Individuals in these societies sometimes behave as if they are not maximising their own fitness but lowering it in order to maximise somebody else's fitness. At the approach of a predator a squirrel gives an alarm call that warns off its neighbours but the individual that gives the alarm call itself attracts the attention of the predator and increases its chances of becoming prey. The grand finale in the evolution of such altruistic behaviour is the case of the worker honey bee that never reproduces on its own but rather spends its entire life-time working for its colony. Nor does this superaltruist hesitate to sting an approaching predator notwithstanding the fact that stinging is suicide. Every honey bee that stings dies within the next few minutes because its barbed sting as well as a part of its intestines are pulled out as it tries to fly away. It was this example of sterile workers in social insects that attracted the attention of Darwin.

\section{The social insects}

Understanding the forces that mould the evolution of social behaviour is one of the most challenging areas of modern biology. A whole new field of sociobiology has developed to meet this challenge (Wilson 1975; Barash 1982). Social insects, especially the ants, bees and wasps have been the focus of special attention in this context (Wilson 1971). The reasons for this have been three fold. Firstly, social insects show the most extreme forms of altruistic behaviour such as the case of the sterile worker bee. Secondly these insects exemplify a series of stages in the course of evolution from the solitary to the highly eusocial. Thirdly the ants, bees and wasps are characterized by a peculiar kind of genetics known as haplodiploidy that introduces asymmetries in genetic relatedness between siblings on the one hand and between parents and offsprings on the other thereby predisposing them towards sociality. (see below).

\section{The theory of kin selection}

\subsection{Statement of the theory}

Hamilton (1964a, b) proposed what has now come to be known as the theory of kin selection. Hamilton argued that social or altruistic traits are selected for by natural 
selection because, although they decrease the classical individual fitness of an animal, they serve to increase the number of copies of the genes coding for such behaviour. This is because altruism is often directed towards genetic relatives who are also likely to carry the same genes. Thus there is really no altruism from the point of view of the genes. Altruism at the level of an individual animal is simply the genes' way of making more copies of itself. Thus animals that behave altruistically may still be maximising their 'inclusive fitness' which is the sum of their direct contribution through their offspring to the gene pool and their indirect contribution through their relatives. This argument can be stated precisely as follows:

As altruistic act will be favoured if

$$
\frac{b}{c}>\frac{1}{r}
$$

where $b$ is the benefit to the recipient, $c$ the cost to the donor and $r$ the coefficient of genetic relatedness between donor and recepient, benefit and cost being measured in fitness units. In other words an altruistic act will be favoured if the benefit to the recipient devalued by the probability that he carried the gene in question is greater than the cost to the donor. Altruistic traits will therefore spread rather easily either if the benefit to cost ratio is high or if the recipient and donor are very closely related.

\subsection{Haplodiploidy}

Consider a hypothetical diploid organism that gives up producing its own offspring and instead helps its parents to produce more of its siblings. This behaviour, if genetically coded, will spread in the population only if our hypothetical organism can raise more siblings than it gives up offspring. This is because both siblings and offsprings are equally related to it $(r=0.5)$. On the other hand if the siblings were more closely related to it than its own offspring, then the behaviour would be selected even if less siblings were produced than offspring given up. This is the kind of situation that occurs in many social insects. The only truly social (with sterile castes) animals are among ants, bees, wasps, termites and a single example from higher animals, the naked mole rat. Of these ants, bees and wasps belong to the insect order Hymenoptera a group characterised by haplodiploidy. Males develop from unfertilized eggs and are consequently haploid. Females develop from fertilized eggs and are diploid. This introduces asymmetries in genetic relatedness (table 1). For example a female hymenopteran is more closely related to her sister $(r=0.75)$ than her own daughter $(r=0.5)$.

Table 1. Coefficients of relatedness under haplodiploidy assuming complete outbreeding.

\begin{tabular}{lcccccc}
\hline & Daughter & Son & Sister & Brother & Mother & Father \\
\hline \multirow{2}{*}{ Female } & 0.5 & 0.5 & $\frac{0.75}{\mathrm{Av}=0.5}$ & 0.5 & 0.5 \\
Male & $\frac{1.0}{\mathrm{Av}=0.5}$ & 0.5 & 0.5 & $\frac{1.0}{\mathrm{Av}=0.5}$ \\
\hline
\end{tabular}




\subsection{Implications of haplodiploidy}

The concept of inclusive fitness together with a knowledge of the asymmetries in genetic relatedness lead to a number of predictions summarised by Wilson (1971) as follows:

(i) True sociality should be more common in haplodiploid organisms than in diploid ones.

(ii) Queens should not be mated by more than one unrelated male.

(iii) Males should be more selfish than females.

(iv) Females should be more altruistic towards their sisters than towards their brothers or neices.

(v) Workers should prefer their own sons over their brothers.

Wilson (1971) also summarised the evidence supporting these predictions and showed that most of the evidence is in qualitative agreement with the theory of kin selection. As pointed out by him this qualitative agreement means that "the factor of haplodiploid bias should be taken into account in future evolutionary interpretations and as a guideline in planning some further empirical research' but not as proof of the correctness of the theory. What then should we do in order to ascertain the validity of kin selection theory? First we ought to put the theory to a critical test by generating quantitative predictions that can potentially falsify the theory and perform the appropriate experiments to see if the predictions are borne out. This alone is sometimes considered inadequate and what we need therefore is also a comparison of the predictions of two or more competing theories (see Lakatos and Musgrave 1970 for a detailed discussion of the methodology of scientific research). In recent years some attempts have been made in providing both these requirements and in the remaining pages some of these studies are reviewed.

\section{Alternative theories}

\subsection{Parental manipulation}

The theory of parental manipulation advanced by Alexander (1974) states that altruistic behaviour could evolve even if it does not increase the inclusive fitness of the altruistic animal because selection can act on the parent to manipulate some of its offspring to be altruistic towards the rest. Ecological conditions could be imagined under which, an animal that produces a certain fraction of sterile offspring which in turn help the remaining fraction of fertile offspring to survive and reproduce better, could leave behind more grandchildren than an animal that produces all fertile, selfish offspring.

\subsection{Mutualism}

Lin and Michener (1972) emphasizing the ecological factors involved in the evolution of altruistic behaviour argue that at least in the early stages of the evolution of sociality, when complete sterility had not yet evolved, mutual advantage in defence against predation for example, might have been an important force. This idea might be more important in present day primitively eusocial insects than has been hitherto suspected (see below). 


\section{Testing the theories}

In this section three major approaches that are being pursued in making quantitative tests of the theories are discussed. The first is the direct measurement of inclusive fitness, the second tests predictions of optimal sex investment strategies and the third involves quantitative studies on the ethology of primitively eusocial insects. I will discuss the logic behind each of these approaches and then very briefly review illustrative examples of empirical investigations representing these approaches, making however no attempt to provide a comprehensive review of the literature. The interested reader may consult several excellent reviews dealing both with theoretical ideas as well as empirical studies on social insects (Evans and West-Eberhard 1970; Wilson 1971; Hamilton 1972; Spradbery 1973; Michener 1974; West-Eberhard 1975; Hermann 1979-82; Starr 1979; Edwards 1980; Jeanne 1980; Barash 1982; Charnov 1982; Michod 1982; Brian 1983).

\subsection{Computation of inclusive fitness}

This approach has mainly been applied to primitively eusocial wasps of the genus Polistes. Typically these wasps follow one of two strategies. Sometimes a single female initiates a colony on her own and raises the first brood of offsprings unaided by any other wasps and later the daughters from her first brood become workers and help her to raise reproductive male and female offsprings. Alternatively a group of females of the same generation, typically sisters, jointly found a nest and again produce a first brood of workers which help them raise reproductive offspring. Here all the cofoundresses do not contribute equally to the production of reproductive offspring. Sometimes only a single dominant $(\alpha)$ female lays all the eggs while the other cofoundresses remain subordinate and behave like workers. Even if the subordinate $(\beta)$ cofoundresses lay some eggs this number is usually less than that laid by the $\alpha$ foundress. In such a situation it is possible to calculate the productivities of the single foundress colonies and the multiple foundress colonies. With a knowledge of the genetic relatedness between cofoundresses and, the exact number of offsprings produced by each cofoundress one can compute the fitness of the solitary foundress as well as the inclusive fitness of the dominant cofoundress and each subordinate foundress in multiple foundress colonies.

For the sake of simplicity let us consider two full sisters $\alpha$ and $\beta$, each having mated with any one male, in a diploid population (table 2). When nesting solitarily (table $2 \mathrm{~A}$ ) let them produce 10 offspring each thus having an individual fitness of 5 (10 offspring $\times 0.5$, the coefficient of genetic relatedness to each offspring) and an inclusive fitness of 7.5 each (individual fitness of $5+$ indirect contribution of 2.5 as a result of 10 neices or nephews $\times 025$, the coefficient of relatedness to each nephew or neice). Note that the term individual fitness is used for the direct contribution to the gene pool and the term inclusive fitness for the sum of the direct and indirect contributions. When they nest together (table 2, B) and if their summed productivity does not increase but merely $\alpha$ produces all the 20 possible offspring and $\beta$ produces none of her own but helps her sister, $\alpha$ has a fitness as well as inclusive fitness of 10 while $\beta$ has a fitness of 0 but an inclusive fitness of 5 . This situation is advantageous to $\alpha$ but not to $\beta$. $\beta$ will therefore not be selected to accept this subordinate role. Nor will selection acting on the parents of $\alpha$ and $\beta$ favour their manipulatine $\beta$ into remaining subordinate and helping $\alpha$. 
Table 2. Conditions for the evolution of sterility in a diploid population where $\alpha$ and $\beta$ are full sisters.

\begin{tabular}{|c|c|c|c|c|}
\hline & & $\alpha$ & $\boldsymbol{\beta}$ & Remarks \\
\hline A & $\begin{array}{l}\text { Number of offspring } \\
\text { Classical individual fitness } \\
\text { Inclusive fitness }\end{array}$ & $\begin{array}{c}10 \\
5 \\
7 \cdot 5\end{array}$ & $\begin{array}{l}10 \\
5 \\
7.5\end{array}$ & $\begin{array}{l}\text { Solitary } \\
\text { Nesting }\end{array}$ \\
\hline B & $\begin{array}{l}\text { Number of offspring } \\
\text { Classical individual fitness } \\
\text { Inclusive fitness }\end{array}$ & $\begin{array}{l}20 \\
10 \\
10\end{array}$ & $\begin{array}{l}0 \\
0 \\
5\end{array}$ & $\begin{array}{l}\text { Sterility } \\
\text { Will not be selected }\end{array}$ \\
\hline C & $\begin{array}{l}\text { Number of offspring } \\
\text { Classical individual fitness } \\
\text { Inclusive fitness }\end{array}$ & $\begin{array}{l}21 \\
10.5 \\
10 \cdot 5\end{array}$ & $\begin{array}{l}0 \\
0 \\
5 \cdot 25\end{array}$ & $\begin{array}{l}\text { Parental } \\
\text { manipulation }\end{array}$ \\
\hline D & $\begin{array}{l}\text { Number of offspring } \\
\text { Classical individual fitness } \\
\text { Inclusive fitness }\end{array}$ & $\begin{array}{l}31 \\
15.5 \\
15 \cdot 5\end{array}$ & $\begin{array}{l}0 \\
0 \\
7 \cdot 75\end{array}$ & Kin selection \\
\hline E & $\begin{array}{l}\text { Number of offspring } \\
\text { Classical individual fitness } \\
\text { Inclusive fitness }\end{array}$ & $\begin{array}{c}21 \text { or } 0 \\
10.5 \text { or } 0 \\
10.5 \text { or } 5 \cdot 25\end{array}$ & $\begin{array}{c}0 \text { or } 21 \\
0 \text { or } 10.5 \\
5.25 \text { or } 10.5\end{array}$ & $\begin{array}{l}\text { Classical } \\
\text { Individual selection }\end{array}$ \\
\hline
\end{tabular}

Sterility on the part of $\beta$ will thus not spread by any of the mechanisms under consideration, parental manipulation, kin selection or individual selection. On the other hand, when nesting jointly (table 2, C), if there is even a slight increase in the total productivity such as $\alpha$ being able to produce 21 offspring then the situation is quite different. Although $\beta$ still has a lower inclusive fitness than when she nested solitarily, $\alpha$ and $\beta$ together have done better than when they were nesting solitarily. Thus it pays the parents of $\alpha$ and $\beta$ to manipulate $\beta$ into being subordinate and sterile because now the parents of $\alpha$ and $\beta$ have more grandchildren. Parental manipulation can thus promote the spread of sterility on the part of $\beta$ although $\beta$ loses in the process. If the productivity increases substantially (table 2, D) such that $\alpha$ produces, say, 31 offspring then $\beta$ gets an inclusive fitness of 7.75 although she does not produce a single offspring. Now kin selection acting on $\beta$ will promote the spread of subordinate behaviour as $\beta$ now contributes more to the gene pool of the population than when she was nesting solitarily and producing 10 of her own offspring. The exact amount by which productivity under joint nesting should increase would depend upon the degree of relatedness between the altruist and those she individual raises. Under haplodiploidy, if a female worker raises sisters in place of daughters then the productivity need not even increase because the workers are more closely related to their sisters than to their daughters $(0.75 v s 0.5)$. If they raise equal numbers of brothers and sisters of course they gain no more fitness than they would, had they raised sons and daughters instead. The asymmetries in genetic relatedness created by haplodiploidy could however be capitalised on if the workers produce more sisters and less brothers. This by itself is a prediction of kin selection theory whose verification has also been attempted (see below).

It is of course possible that both $\alpha$ and $\beta$ produce offspring when nesting jointly and 
that both produce more offsprings than when they were nesting solitarily. This will lead to the evolution of joint nesting by classical individual selection but there will be no sterility. Such a situation is envisaged by Lin and Michener (1972) in the early stages of the evolution of sociality. Ecological conditions can be sufficiently harsh and it may for example be impossible for a single female to guard her nest against predators as well as forage for food. Thus by nesting jointly both $\alpha$ and $\beta$ may increase their offspring production. Once such joint nesting gets established in the population it could give scope for the evolution of parental manipulation or kin selection. Another interesting case of evolution of sterility is possible through classical individual selection (mutualism) which may be more important in present day primitively eusocial insects. For example, let $\alpha$ and $\beta$ nest jointly but who will lay the eggs and who will remain sterile be decided by chance (table 2, E). If $\alpha$ and $\beta$ each have on the average an equal chance of egg laying then, even if there is a slight increase in productivity due to joint nesting, classical individual selection will promote such sterility. When nesting, solitarily $\alpha$ and $\beta$ each get an inclusive fitness of 7.5. Here each gets on the average $10 \cdot 5+5 \cdot 25=15 \cdot 75 / 2=7 \cdot 875$. In Polistes exclamans the $\beta$ foundress remains subordinate and waits for the $\alpha$ foundress to produce a batch of workers. When it is time to lay eggs that will mature into reproductives the $\beta$ foundress suddenly becomes very aggressive and challenges the $\alpha$ foundress. In a substantial number of cases the $\beta$ foundress succeeds in driving away the $\alpha$ foundress and then lays all the eggs. (Alan MacCormac personal communication). This would be a situation analogous to the example described above and here subordinate behaviour and sterility with a certain probability could be brought about by classical individual selection.

Perhaps the most complete study attempting to distinguish between different theories by the computation of inclusive fitness is that of Metcalf and Whitt (1977) on Polistes metricus. They used enzyme electrophoresis to determine the genetic relatedness between different individuals in the colony and knowing the productivity of single and multiple foundress colonies they were able to calculate the inclusive fitnesses of a solitary foundress, the dominant $\alpha$ foundresses and the subordinate $\beta$ foundresses of joint nesting colonies. Their results are as follows:

In the population studied by Metcalf and Whitt, $83 \%$ of the colonies were solitary foundress colonies. In multiple foundress colonies, $\alpha$ foundresses had a relative inclusive fitness $1.83 \pm 0.57$ times that of a solitary foundress while the $\beta$ foundress had a relative inclusive fitness $1.39 \pm 0.44$ times that of a solitary foundress. A female's expected number of grandchildren from two of her daughters jointly founding a nest is 1.55 times what she would achieve by the two daughters acting as two solitary foundresses.

Since the $\beta$ foundress does not have an inclusive fitness significantly greater than that of the solitary foundress, neither kin selection theory nor mutualism (classical individual selection; table 2, E) can be accepted as the selective force responsible for the altruistic behaviour of $\beta$ although the authors themselves conclude that their results are in accordance with the predictions of kin selection theory. Parental manipulation could explain this behaviour on the part of $\beta$ provided the figure of ' 1.55 times more grandchildren' is statistically significant (no standard deviation is provided by the authors). Besides one does not know how to interpret the fact that $83 \%$ of the colonies were single foundress colonies (see below). A very similar study was conducted on a related species $P$. fuscatus (Noonan 1981). Not having used electrophoresis Noonan did not know the exact values of genetic relatedness but made her computations of inclusive fitness for different possible values of relatedness between the dominant and 
subordinate females on a nest (full sisters, half sisters etc.). These results showed that subordinate females lay some eggs (and gain some individual fitness) but not enough to make subordinate roles better than solitary nesting. Because foundresses are ofte: sisters the subordinates have inclusive fitness values greater than their individual fitness values. Noonan's data however do not permit distinction between parental manipulation and kin selection models because of the high variances associated with her mean values of inclusive fitness. Thus we find that the best empirical studies using the approach of computing inclusive fitness does not permit us to draw any definite conclusions.

I would also like to argue that there are difficultiès with this approach itself. Firstly the kind of analysis described above compared wasps which are naturally nesting either solitarily or jointly. Implicit in such a comparison is the assumption that the two females being compared are reproductively equivalent and that any difference in productivity is only because of solitary versus joint nesting. We have no information that might help decide whether this assumption is valid or not. However, if solitary and joint nesting females have different reproductive potentialities, then the effects of the intrinsic differences in productivity and the effects of nesting strategy will be confounded in the analysis. The second problem with this approach is that the frequency of solitary versus joint nesting varies widely in different situations. No attempt has so far been made to take this into consideration. Ideally, the theory should be able to predict the frequency distribution of foundress size associations. Even if we find that the inclusive fitness of the subordinate foundress is much higher than a solitary foundress, there would still remain a puzzle if it turns out that most of the females prefer to nest solitarily.

\subsection{Sex investment ratios}

We argued earlier that haplodiploidy predisposes the hymenopterans to the path of sociality because a female is more closely related to her sister than to her daughter. But this asymmetry exactly cancels out because a female is less closely related to her brother than to her son. Her average relationship to her offspring of 0.5 is exactly the same as her average relationship to her siblings $\left(\frac{0.75+0.25}{2}\right)$. Trivers and Hare (1976) argued therefore that workers in hymenopteran societies should be capitalizing on the asymmetries created by haplodiploidy by investing differentially in their sisters and brothers, in fact in the ratio $3: 1$, which is the ratio of their genetic relatedness to their sisters and brothers respectively. Since it is the workers who feed the larvae, it should be easy enough for them to feed their sisters and brothers differently and achieve the maximum possible inclusive fitness. Notice however that on the Fisherian argument (Fisher 1930) the mother queen who is equally related to her sons and daughters would prefer an equal investment in brood of the two sexes (assuming on outbreeding, random mating population). In other words there is a conflict of interests between the queen (who prefers a 3:1 ratio of investment) and the workers (who prefer a 3:1 ratio of investment). Notice that this approach too.makes a precise quantitative prediction and also contrasts kin selection theory with the parental manipulation theory. If the parental manipulation theory is correct, then it means that the mother queen should be able to have her way and manipulate the workers to invest equally in the two sexes although it is not the optimum strategy for them. If kin selection theory on the other hand is 

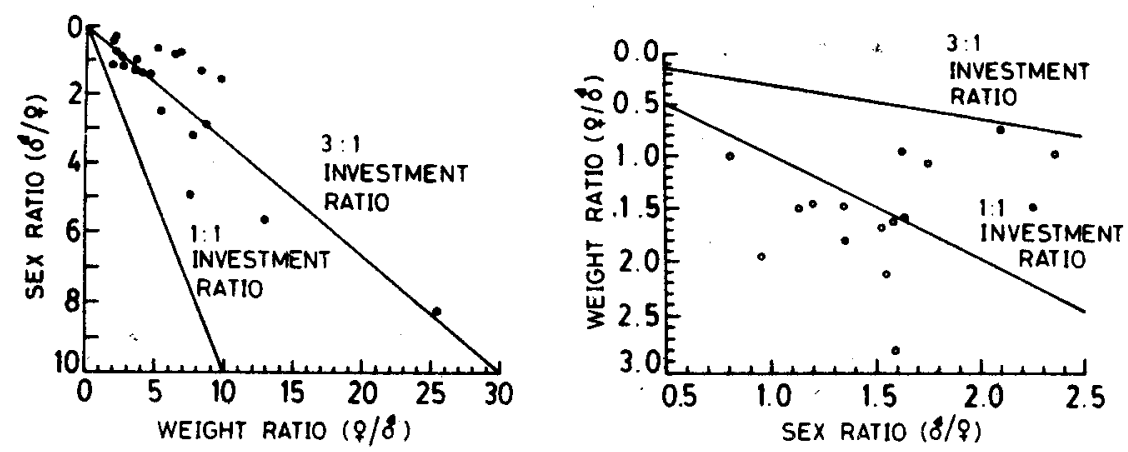

Figure 1. A. Relationship between the sex ratio and weight ratio of reproductive caste ants. The two lines show $1: 1$ and 3:1 investment ratios. B. Relationship between sex and weight ratios for solitary bees (open circles) and solitary wasps (closed circles) compared for $1: 1$ and 3:1 investment ratios (modified slightly from Trivers and Hare 1976, after Barash 1982. Reprinted with permission).

correct, then workers behave altruistically only because this is the strategy that snaximises their inclusive fitness. But it would not maximise their inclusive fitness if they invested equally in their brothers and sisters. Workers should therefore have their way and invest in the ratio $3: 1$.

Trivers and Hare (1976) weighed the total male and female reproductive brood in a large number of species and appeared to provide overwhelming support for kin selection theory. In a large number of monogynous ant species the weight ratio of the reproductive brood was significantly close to the $3: 1$ prediction. Besides, in solitary bees and wasps, and termites the investment ratio as expected was close to $1: 1$.

Soon after the publication of this study, Alexander and Shermann (1977) pointed out that there are serious problems with the interpretations of Trivers and Hare. The predictions used by Trivers and Hare are valid only if the queens have mated only once and only if the populations are completely outbreeding. If the queens mate more than once then the workers are not necessarily full sisters of the reproductive siblings and therefore they would not be expected to invest in the ratio $3: 1$. If the population inbreeds then according to the theory of local mate competition proposed by Hamilton (1967) even the queens would prefer a female biased sex investment ratio. According to Alexander and Shermann, Trivers and Hare's data thus may not represent the triumph of kin selection theory over parental manipulation theory but may simply be a response to local mate competition. Supporting this argument is a 1:1 ratio of investment demonstrated in Polistes fuscatus in a situation where local mate competition was known to be absent (Noonan 1978). One.obvious difficulty here is the lack of good empirical data on multiple mating and local mate competition. Multiple mating has long been realised to be very common in social insects (Wilson 1971) but it has often been ignored because the sperms from different males have been assumed not to mix in the spermatheca (Orlove 1975, for example). Page and Metcalf (1982) have recently demonstrated that at least in the honey bee, sperms from different males do mix and that the average genetic relatedness between workers and the reproductive sisters they rear can be quite low. In any case it is clear that multiple mating and local mate competition both influence the predictions of optimum sex investment ratios in the framework of kin selection theory and parental manipulation theory. Information on the extent of multiple mating and local mate competition in social insects is thus urgently needed. 
Given certain levels of multiple mating and local mate competition, the prediction of optimum sex investment ratios by kin selection theory and parental manipulation theory would be different from those used by Trivers and Hare. Recently Joshi and Gadagkar (1985) have modelled this phenomenon and computed the optimum sex investment ratios under different levels of multiple mating and local mate competition (figure 2). This has been done by considering a haplodiploid population with monogynous colonies where a certain fraction of the reproductive offspring disperse and outbreed while the remaining fraction undergo brother-sister mating. Our results can be summarised as follows (figure 2). In the absence of multiple mating and local mate competition, parental manipulation theory (queen control) predicts a $1: 1$ investment ratio while kin selection theory predicts a $3: 1$ ratio (female: male). Local mate competition biases the investment ratio in favour of females in the frame work of both the theories. For any given value of local mate competition however, the investment ratio predicted by parental manipulation theory is more male biased than that predicted by kin selection theory. Multiple mating does not affect the prediction of the parental manipulation theory at all but makes the optimum investment ratio under kin selection theory more male biased than the single mating case. We hope that the exact predictions of sex investment ratios in the framework of these two theories provided by us will be used in a fresh attempt to distinguish between these theories in future studies that should attempt to measure not only the sex investment ratios but also the levels of multiple mating and local mate competition.

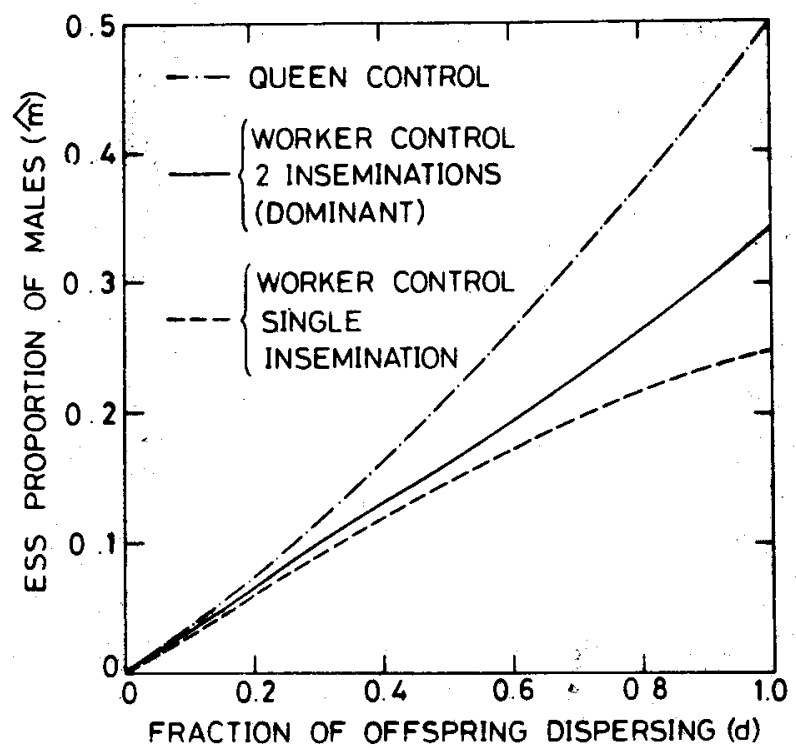

Figure 2. Optimum sex ratio at different levels of local mate competition. Evolutionarily stable proportions of male offspring $(\mathrm{m})$ are plotted as a function of the fraction of offspring dispersing to outbreed (d) for three cases. (_- - when queens control the ratio of investment (parental manipulation). (- - ). when workers control the ratio of investment but the queen has mated only once i.e., the workers are rearing their brothers and full sisters. $(-)$ workers control the ratio of investment but the queen has mated with two unrelated males so that, the workers are rearing their brothers and a combination of full and half sisters. In the model investigated, the workers cannot distinguish between their full and half sisters. (From Joshi and Gadagkar 1985. Reprinted with permission.) 


\subsection{Quantitative ethology}

In the last few years I have been studying the behaviour of adults on colonies of primitively eusocial wasps such as Ropalidia in India and Polistes in America with the hope that these studies will in the long run provide a third approach to understanding the forces that mould the evolution of social behaviour (Gadagkar 1980; Gadagkar and Joshi 1982a, b, 1983, 1984, 1985). Taking Ropalidia marginata in India as an example I shall now illustrate this approach. Colonies of Ropalidia marginata are initiated by one or a group of females (foundresses) at any time of the year (Gadagkar et al 1982a,b). One of these foundresses assumes the role of the queen while the others remain subordinate to the queen and assume the role of workers. Of the female offspring produced in such a colony many remain at the parent colony and become workers although some leave it to found or join other colonies. Males disappear from the colony within a few days after their emergence. The lack of a very severe winter in peninsular India permits these colonies to be perennial. A single colony can therefore survive for many years. This makes it possible for queens to be replaced (Gadagkar unpublished observations). When an existing queen dies or is driven away, one of the other females takes over and begins to lay eggs. In addition, females sometimes leave their parent colonies to found their own colonies either alone or in small groups where one of them again becomes the queen. These events can occur any time in the year and therefore every female must have a fair chance of becoming a queen.

I argue that in such a situation these colonies cannot simply consist of a queen and a bunch of willing workers but each colony must be a highly competitive association of female wasps each trying to maximise its chances of becoming a queen either by leaving the colony at the appropriate time and perhaps with the appropriate company or to challenge the existing queen at the appropriate moment and inherit the nest, its brood and the workers. Using two monogynous colonies of $R$. marginata, I sought to discern the competitive strategies of these wasps by carefully studying and quantifying their behaviour. Instead of following the classical method of concentrating either on species specific behaviour patterns or on certain kinds of behaviours that appear to be important from our point of view, I decided to study the patterns in which the wasps allocated their time between different behaviours. Since time must be a very limiting resource, I considered those behaviours important in which the wasps spent more time. Besides, my emphasis was on the differences between different individuals within a colony. Sitting, sitting with raised antennae (alert to external disturbance), sitting with raised antennae and raised wings (a state of alarm), walking on the nest, being in the cells, and being away from the nest turned out to be the activities in which the wasps spent most of their daylight hours. For these six behaviours I constructed time-activity budgets for a number of individually identified animals. As it turns out, all animals studied spent about $95 \%(95.9 \pm 4.4)$ of their daylight hours in these six behaviours but the manner in which they allocated their time between these behaviours was highly variable (figure 3 ).

These time-activity budgets were analysed by multivariate statistical techniques such as principal components analysis and hierarchical cluster analysis with the aim of understanding the difference between individuals. The wasps could be classified into three distinct behavioural castes (figure 4) with sitting, sitting with raised antennae and being absent from the nest as the main attributes of the three clusters respectively (figure 5). I had also collected data on the frequencies of certain rare behaviours such as 

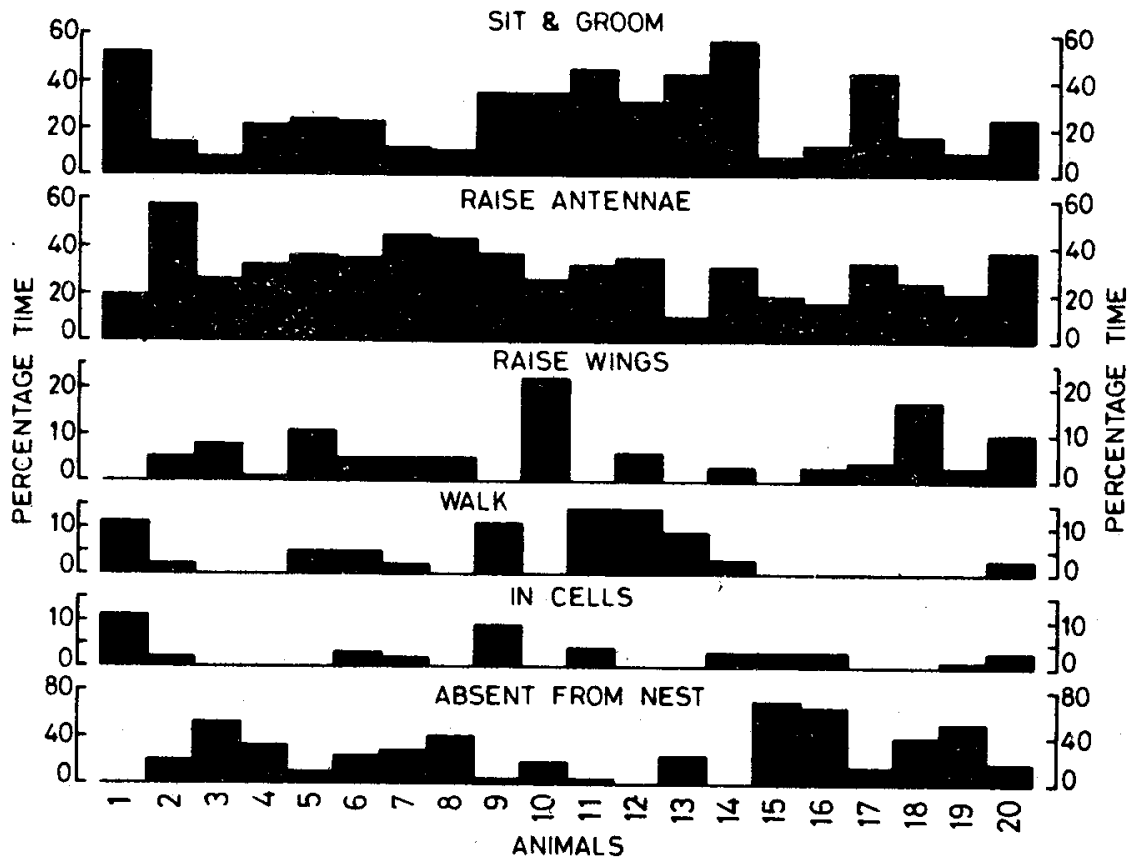

Figure 3. Time-activity budgets of 20 individually identified animals from 2 monogynous nests for 6 behaviours. Animals 1 and 14 are the queens of nest 1 and 2 respectively.

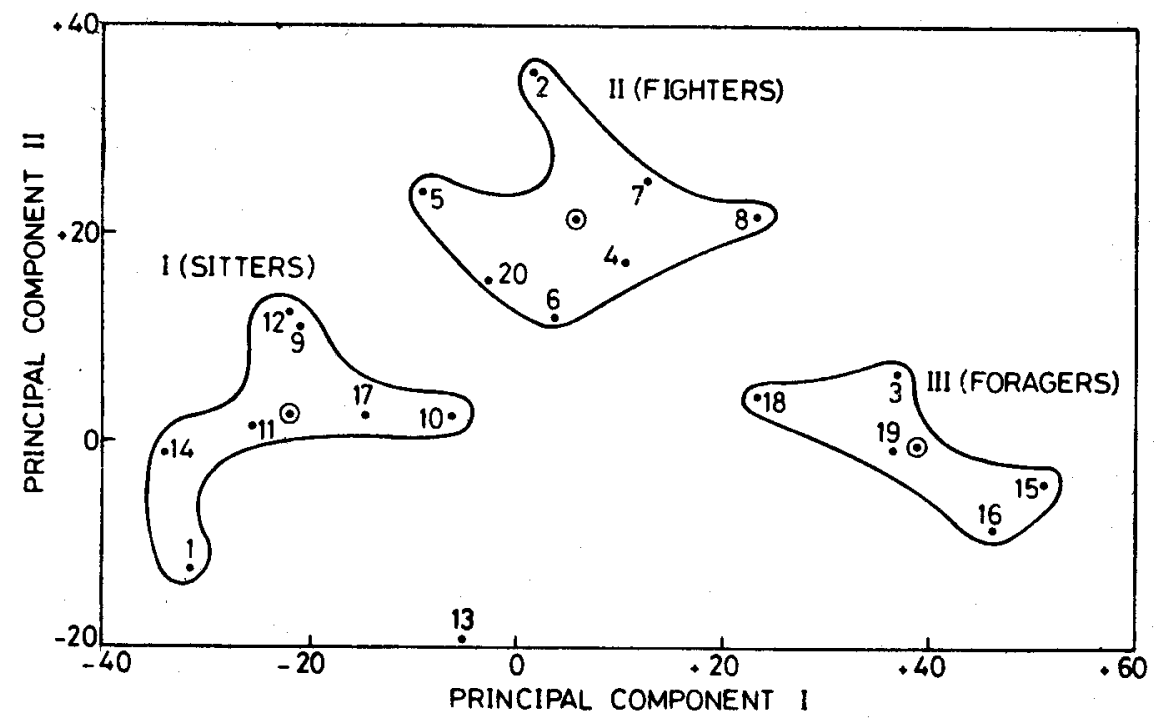

Figure 4. Behavioural castes of $R$. marginata. Twenty wasps from 2 different nests are shown as points in the coordinate space of the amplitudes associated with the first two principal components. The points fall into three clusters (or castes) by the criterion of nearest centroid. Circled dot $=$ centroid. (From Gadagkar and Joshi 1983a. Reprinted with permission). 


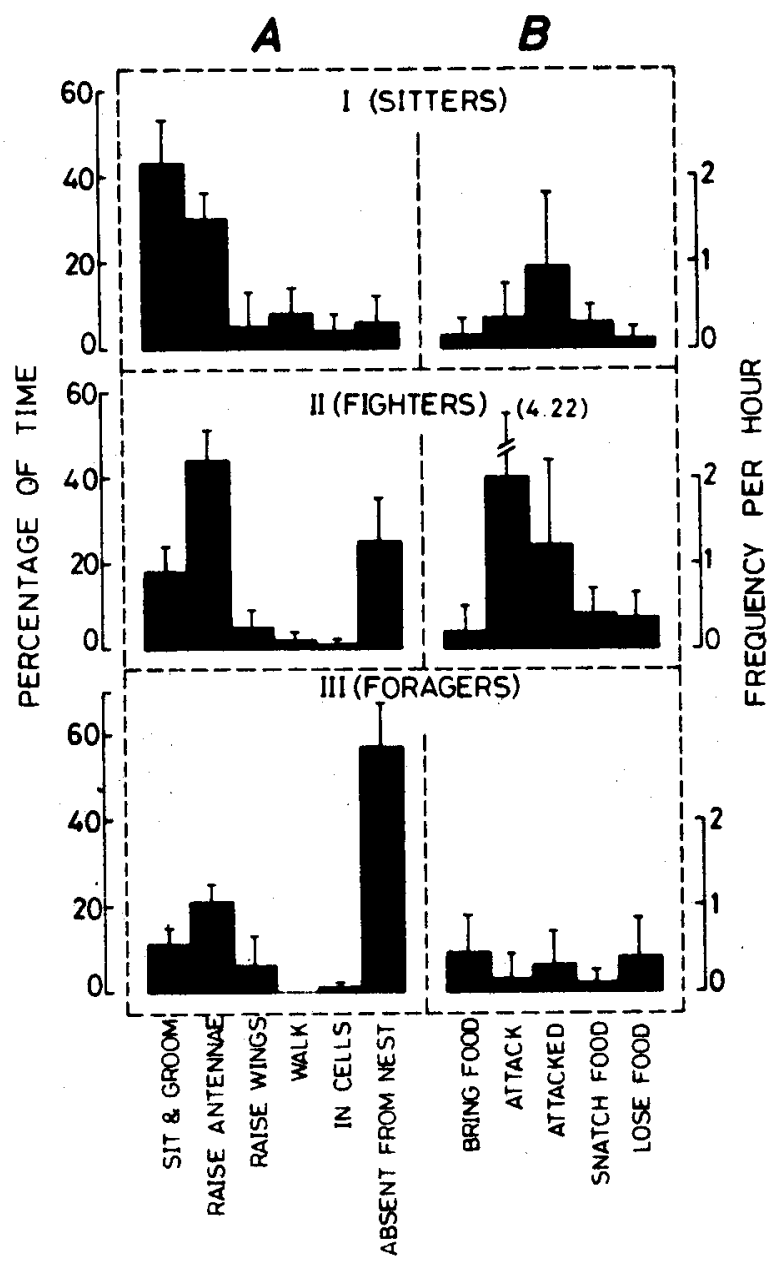

Figure 5. Mean behavioural profiles of the clusters obtained in figure $4 \mathrm{~A}$, mean percentages of time spent in each of the six activities that were used in obtaining the clusters are shown for sitters, fighters and foragers. B. mean frequencies per hour of the five activities that were not used to obtain the clusters are shown for sitters, fighters and foragers. (From Gadagkar and Joshi 1983a. Reprinted with permission).

dominance behaviour, egg laying etc. which were not used in the analysis. Sitting with raised antennae is positively correlated with dominance behaviour $(P<0.01)$. Wasps absent from the nest often return with food loads (figure 5B). The three clusters were thus named Sitters, Fighters and Foragers (although it is possible that wasps absent from their nests spent some of their time looking for other nests to join or new nest sites to start their own nests).

We can now begin to interpret the biological significance of this behavioural caste differentiation. Note that the two queens (individuals 1 and 14) are among sitters (queens are recognised by their egg laying behaviour). Thus the queens do little other than sitting and grooming because it is perhaps the best strategy to conserve their 
energy and maximise their egg laying capacity. But this also means that the queens face little competition from their nestmates who can therefore be 'trusted' to do all the work for the colony. This seemed to be the situation in the colonies of $R$. marginata that were studied. Significantly when some polygynous colonies of another species $R$. cyathiformis were studied, the queens were not sitters but fighters. In the latter case the queens do not just sit but they were the most active individuals in the colony. In $R$. marginata however, there were many sitters who were not queens. These we hypothesise are 'hopeful queens' who still have some chances of becoming queens. On the other hand the fighters could also be hopeful queens. who are following an alternative strategy of maximising their chances of becoming queens. These hypotheses assume significance because they can be readily tested. Experiments are in progress where I am studying a number of colonies, classifying the wasps and then removing the queens to see who takes over. Take over somebody does, but preliminary results indicate that either a Sitter or a Fighter could be the replacement queen (Gadagkar unpublished observations). The foragers are interpreted as having the least chance of becoming replacement queens. This interpretation holds whether the foragers really spent all their time away from the nest in foraging or attempted to join or initiate new nests. The fighters very frequently behave dominantly towards other members of the colony. Apart from establishing their claim to the position of the next queen this fighting could have other functions. For example it may serve to keep the foragers active. I have often seen foragers leave the colony after a series of attacks by a dominant wasp. That the queens could be sitters or fighters depending on the extent of reproductive competition they face from their nest mates seems to be supported by our results. As mentioned above queens of monogynous colonies of $R$. marginata were sitters while queens of polygynous colonies of $R$. cyathiformis were fighters. Even in $R$. cyathiformis when a single foundress was attending a colony and thus had no one to compete with, she was a Sitter i.e. she did not spend more time with raised antennae. Furthermore in Polistes versicolor in Panama, I had an opportunity to study both pre-emergence colonies as well as post emergence colonies. Pre-emergence colonies are associations of females of the same generation. In such a situation, the closest possible relationship between the workers and the brood they rear is $3 / 8$ (neices or nephews). These colonies are therefore likely to be much more competitive than post-emergence colonies consisting of a mother queen and her daughter workers; the latter caring for their siblings. The queens in two pre-emergence colonies studied were Fighters while the queen in the postemergence colony studied was a Sitter (Gadagkar and Joshi in preparation).

The behaviour of the wasps is very highly variable and seemingly unpredictable. But I believe that in this variability lies the clue to the understanding of the evolution of sociality. Wasps that all look morphologically similar may behave as sitters, fighters or foragers, they may be queens or subordinate workers, they may challenge an existing queen and chase her away to take her position, they may leave the colony alone or with a submissive group to start new colonies. Kin selection theory predicts that the wasps should behave so as to maximise their inclusive fitness. Parental manipulation would predict that the animals should at least sometimes behave not according to their own optimum criteria' but according to what is best for their parents to produce the largest number of grandchildren. If we know what factors influence the chances of a wasp becoming an egg layer in a given circumstance it should be possible to predict the behaviour of a given animal within the framework of a particular theory. With this long term aim in mind experiments are in progress in my laboratory to determine the relative 
contributions of body size, age, prior social experience, hormone levels etc. to the egg laying capacity and the capacity to win in an encounter with a conspecific.

It is in the context of such present day primitive insect societies that $I$ have been discussing above that mutualism or classical individual selection of the kind considered in table $2 \mathrm{E}$ might be important. For instance a wasp may stay on at the parent colony and work as a subordinate individual if it has a fair chance of becoming the next queen. There may be a certain probability of not succeeding but if more inclusive fitness is gained on the average by taking the risk than by solitary nesting, classical individual selection will favour this behaviour and we will see certain individuals remaining sterile even if that particular individual does not have higher inclusive fitness than its solitary counterpart.

\section{Conclusions}

In conclusion it may be said that today we have a very attractive body of theoretical ideas concerning the forces that might be responsible for the origin and maintenance of social behaviour especially in social insects. Several simple minded attempts have been made to test these theoretical ideas but none has yielded unambiguous results. This is primarily because the social insects live in rather complex societies and pursue complex strategies. We need much more information on the details of the lives of these insects, especially in areas such as breeding structure of populations and the factors that influence the chances of an animal's success in an encounter with a conspecific before we can launch more sophisticated empirical tests of the theories.

\section{Acknowledgements}

It is a pleasure to thank Madhav Gadgil, N V Joshi, Mary Jane West-Eberhard, Charles D Michener and V Nanjundiah for reviewing an earlier draft of this paper and W D Hamilton with whom many of the ideas presented in this paper were discussed. My own research discussed here has been supported in part by a grant from the DST, Government of India.

\section{References}

Alexander R D 1974 The evolution of social behaviour Ann. Rev. Ecol. Syst. 5 325-383

Alexander R D and Sharmann P L 1977 Local mate competition and parental investment in social insects; Science $196494-500$

Barash D P 1982 Sociobiology and behaviour (New York: Elsevier) pp. xiv +426

Brian M V 1983 Social insects (London: Chapman and Hall)

Charnov E L 1982 The theory of sex allocation (New Jersey: Princeton Univ. Press)

Darwin C 1859 On the origin of species by means of nat ural selection or the preservation of favoured races in the struggle for life (London: John Murray) ix + 502 pp.

Evans H E and West-Eberhard M J 1970 The wasps (Ann Arbor: Univ. of Michigan Press)

Edwards R 1980 Social wasps; Their biology and control East Grinstead W Sussex England Rentokil

Gadagkar R 1980 Dominance hierarchy and division of labour in the social wasp Ropalidia marginata (Lep.) (Hymenoptera: Vespidae); Curr. Sci. $49772-775$

Gadagkar R, Gadgil M and Mahabal A S :982a Observations on population ecology and sociobiology of the 
paper wasp Ropalidia marginata (Lep.) (Family Vespidae); Proc. Symp. Ecol. Anim. Pop. Zool. Surv. India Part 4 pp. 49-61

Gadagkar R, Gadgil M, Joshi N V and Mahabal A S 1982b Observations on the natural history and population ecology of the social wasp Ropalidia marginata (Lep.) from Peninsular India (Hymenoptera: Vespidae); Proc. Indian Acad. Sci. (Anim. Sci.) 91 539-552

Gadagkar R and Joshi N V 1982a Behaviour of the Indian social wasp Ropalidia cyathiformis (Fab.) on a nest of separate combs (Hymenoptera: Vespidae); J. Zool. London 198 27-37

Gadagkar R and Joshi N V $1982 \mathrm{~b}$ A comparative study of social structure in colonies of Ropalidia. In The biology of social insects (ed.) M D Breed, C D Michener and M E Evans Proc. IX Cong. Int. Union for the study of social insects Boulder, Colorado, usA, August 1982 (Boulder: Westview Press) pp. 187-191

Gadagkar R and Joshi N V 1983 Quantitative ethology of social wasps: Time-activity budgets and caste differentiation in Ropalidia marginata (Lep.) (Hymenoptera: Vespidae); Anim. Behav. 31 26-31

Gadagkar R and Joshi N V 1984 Social organisation in the Indian wasp Ropalidia cyathiformis (Fab.) (Hymenoptera: Vespidae); Z. Tierpsychol 64 15-32

Gadagkar R and Joshi N V 1985 Colony fission in a social wasp; Curr. Sci. 54 57-62

Hamilton W D 1964a The genetical evolution of social behaviour I. J. Theor. Biol. 7 1-16

Hamilton W D 1964b The genetical evolution of social behaviour II. J. Theor. Biol. 7 17-52

Hamilton W D 1967 Extraordinary sex ratios; Science $156477-488$

Hamilton W D 1972 Altruism and related phenomena mainly in social insects; Ann. Rev. Ecol. Syst. $3192-232$

Hermann H R 1979-82 Social Insects (New York and London: Academic Press) Vols I-IV

Jeanne R L 1980 Evolution of social behaviour in Vespidae; Ann. Rev. Entomol. 25 371-396

Joshi N V and Gadagkar R 1985 Evolution of sex ratios in social hymenoptera: Kin selection local mate competition polyandry and kin recognition J. Genet. 64 41-58

Lakatos I and Musgrave A 1970 Criticism and the growth of knowledge (Cambridge: Cambridge University Press) pp. 282

Lin N and Michener C D 1972 Evolution of sociality in insects; Q. Rev. Biol. 47 131-159

Michener C D 1974 The social behaviour of the bees (Cambridge Mass: Harvard Univ. Press)

Metcalf R A and Whitt G S 1977 Relative inclusive fitness in the social wasp Polistes metricus, Beh. Ecol. Sociobiol. 2 353-360

Michod R E 1982 The theory of kin selection; Ann. Rev. Ecol. Syst. 13 23-55

Noonan K M 1978 Sex ratio of parental investment in colonies of the social wasps Polistes fuscatus; Science 199 1354-1356

Noonan K M 1981 Individual strategies of inclusive fitness maximizing in Polistes fuscatus foundresses. In Natural selection and social behaviour (eds) R D Alexander and D W Tinkle (New York: Chiron Press) $18-44$

Orlove M J 1975 A model of kin selection not involving coefficients of relationships; J. Theor. Biol. 49 289-310

Page R E and Metcalf R A 1982 Multiple mating, sperm utilization and social evolution; Am. Nat. 119 263-281

Spradbery J P 1973 Wasps: An account of the biology and nat ural history of solitary and social wasps (Seattle: Univ. Washington Press)

Starr C K 1979 Origin and Evolution of Insect Sociality A review of modern theory. In Social insects (ed.) H R Hermann (New York and London: Academic Press) Vol I 35-79

Trivers R L and Hare H 1976 Haplodiploidy and the evolution of the social insects; Science 191 249-263

West-Eberhard M J 1975 The evolution of social behaviour by kin selection; Q. Rev. Biol: 50 1-34

Wilson EO 1971 The insect societies (Cambridge Messachusetts: Bellknap Press of Harvard University Press) $\mathrm{x}+548$ pp.

Wilson E O 1975 Sociobiology (Cambridge Massachusetts: Harvard University. Press) ix + 697 pp. 